\title{
Fluorescent Cell Staining Methods for Living Hypsibius exemplaris Embryos
}

\author{
Kristen M. McGreevy, ${ }^{1}$ Kira L. Heikes, ${ }^{1,2}$ Shiri Kult, ${ }^{3}$ Marla E. Tharp, ${ }^{3}$ and Bob Goldstein ${ }^{1,2,4,5}$ \\ ${ }^{1}$ Biology Department, University of North Carolina at Chapel Hill, Chapel Hill, North Carolina 27599; \\ ${ }^{2}$ Curriculum in Genetics and Molecular Biology, University of North Carolina at Chapel Hill, Chapel Hill, \\ North Carolina 27599; ${ }^{3}$ Embryology Course, Marine Biological Laboratory, Woods Hole, Massachusetts 02543; \\ ${ }^{4}$ Lineberger Comprehensive Cancer Center, University of North Carolina at Chapel Hill, Chapel Hill, \\ North Carolina 27599
}

The tardigrade Hypsibius exemplaris was chosen as a model system in part because animals and embryos are optically clear at all stages, facilitating the visualization of events in living material. Here we report new methods for introducing fluorescent dyes into developing $H$. exemplaris embryos, including methods for fluorescently marking mitochondria, lysosomes, membranes, and nuclei. The development of these techniques suggests approaches for attempting to introduce other molecules into embryos.

It is essential that you consult the appropriate Material Safety Data Sheets and your institution's Environmental Health and Safety Office for proper handling of equipment and hazardous material used in this protocol.

RECIPES: Please see the end of this protocol for recipes indicated by $<R>$. Additional recipes can be found online at http://cshprotocols.cshlp.org/site/recipes.

Fed cultures of tardigrades See Protocol: Laboratory Culture of Hypsibius exemplaris (McNuff 2018).

Fluorescent organelle stains (see Steps 5 and 10)

Acridine orange staining solution $(1 \mu \mathrm{M})<\mathrm{R}>$ (for lysosomes)

This is a live-cell dye that fluoresces at different wavelengths depending on binding, concentration, and time elapsed: It emits green light (excitation peak, $500 \mathrm{~nm}$; emission peak, $526 \mathrm{~nm}$ ) when bound to dsDNA or acidic compartments, and emits red light (excitation peak, $460 \mathrm{~nm}$; emission peak, $650 \mathrm{~nm}$ ) when bound to ssDNA or RNA. When accumulated in vesicles, excitation with blue light can result in vesicle lysing (Lin et al. 2017).

FM 4-64 staining solution $(10 \mu \mathrm{g} / \mathrm{mL})<\mathrm{R}>$ (for membranes)

This is a lipophilic, red fluorescent, live-cell membrane dye (excitation peak, $515 \mathrm{~nm}$; emission peak, $640 \mathrm{~nm}$ ). The manufacturer recommends using this at concentrations around $5 \mu \mathrm{g} / \mathrm{mL}$; however, we use it at a concentration of $10 \mu \mathrm{g} / \mathrm{mL}$.

LysoTracker Green DND-26 staining solution $(75 \mathrm{~nm})<\mathrm{R}>$ (for lysosomes)

This is a green fluorescent dye that stains acidic compartments in live cells (excitation peak, $504 \mathrm{~nm}$; emission peak, $511 \mathrm{~nm}$ ).

${ }^{5}$ Correspondence: bobg@unc.edu

From the Emerging Model Organisms collection.

(C) 2018 Cold Spring Harbor Laboratory Press

Cite this protocol as Cold Spring Harb Protoc; doi:10.1101/pdb.prot106021 
Equipment

Aspirator tube assembly for microcapillary tubes (Sigma-Aldrich A5177)

Electroporation cuvettes $(0.4 \mathrm{~cm})$ (e.g., Invitrogen P46050) (for marking nuclei)

Gene Pulser and Pulse Controller (Bio-Rad 165-2098) (for marking nuclei)

Glass beads (31- $\mu$ m diameter; Whitehouse Scientific MS0031)

Glass microcapillary tube (e.g., Sigma-Aldrich P0674)

Glass slides and coverslips $(18 \times 18 \mathrm{~mm}, \# 1.5)$

Paintbrush

Paper clip or binder clip (see Step 1)

Pipette puller or flame

Plastic Petri dishes (3.5-cm diameter or larger)

Polystyrene 96-well microplates (e.g., Sigma-Aldrich CLS3799) can be used as an alternative for staining (Step 5).

Spinning disk confocal microscope, equipped for recording video

Stereomicroscope

Syringe needles ( $25 \mathrm{G} \times 5 / 8$ inch; e.g., BD PrecisionGlide 305122)

Test tubes

The fluorescent dye procedures below use the same collection and mounting setup for each dye, but different methods for preparing each dye solution and for introducing the dyes into the embryos. For double-labeling two different structures, we use dyes with nonoverlapping emission spectra. An example is shown in Figure 1. See the Discussion section for limitations and considerations for introducing other molecules.

\section{Collect Embryos}

H. exemplaris mothers lay embryos into their own cuticles, and the adults molt soon thereafter, leaving embryos behind inside the exuviae (shed cuticles). The exuviae can prevent dye from reaching the embryos and therefore should be carefully cut away using a syringe needle (Step 4).

1. Prepare an aspirator pipette by pulling a glass microcapillary tube over a flame or in a needle puller, breaking the tapered end off to leave an opening that is $\sim 0.5 \mathrm{~mm}$ wide, and inserting the blunt end of the tube into an aspirator tube assembly. Close the end of aspirator assembly's tubing furthest from the pipette by folding it over and clipping it with a paper clip or binder clip. Pinch the tubing between a finger and a thumb to control aspiration.

This aspirator pipette can be used to pick up and deposit individual animals or exuviae. Aspiration is controlled by pinching the tubing between a finger and thumb. Aspirator pipettes were traditionally controlled by mouth pipetting, but this method is disallowed by many modern safety protocols. 
K.M. McGreevy et al.

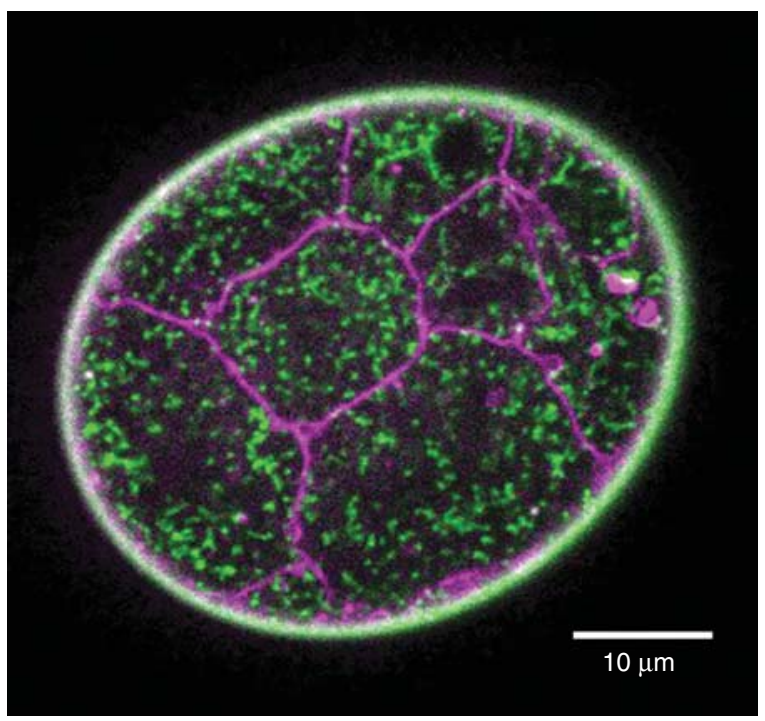

FIGURE 1. Hypsibius exemplaris embryo with mitochondria labeled using Mitotracker Green (in green) and membranes labeled using FM 4-64 (in magenta).

2. Examine fed cultures for embryos at the stages of interest under a stereomicroscope. To collect 1- to 4-cell stage embryos (newly laid), identify tardigrades that are sharing their cuticles with embryos.

The mothers generally exit the cuticle within a few hours after laying.

3. Fill a plastic Petri dish halfway with spring water. Transfer the laying mothers and exuviae with the aspirator pipette to the plastic Petri dish with spring water.

Minimize the transfer of algae, as their autofluorescence interferes with imaging. Remove algal cells from the Petri dish using the aspirator pipette if necessary.

4. Use a syringe needle to carefully cut away the exuviae at an end near the embryos to isolate the embryos from the mother and exuviae.

Dexterity is required to not destroy the embryos. If an exuvium remains attached to embryos, carefully roll it along the bottom of the Petri dish to separate.

Proceed to either Step 5 or Step 10.

Mark Lysosomes, Mitochondria, or Membranes by Soaking

LysoTracker Green and acridine orange can be used in this soaking protocol to mark lysosomes, and MitoTracker Green to mark mitochondria. We have confirmed that LysoTracker Green and acridine orange produce similar staining patterns, and that double-staining with both dyes results in the same components marked with each dye, suggesting that the structures marked by these dyes are likely to be lysosomes as expected.

5. Prepare staining solution from the appropriate stock solution. Pipette $12-15 \mu \mathrm{L}$ of staining solution onto a plastic Petri dish.

To label two cellular compartments at the same time, prepare each dye at double the usual concentration. Mix 6-7.5 $\mu \mathrm{L}$ of each dye into a single droplet.

6. Using an aspirator pipette, transfer 5-15 isolated embryos to the dye droplet.

Twelve to $15 \mu \mathrm{L}$ of dye solution is sufficient for staining 5-15 embryos at a time. Transfer as little water as possible with the aspirator pipette to minimize dilution of the dye solution.

7. Remove the embryos from light and soak the embryos in dye solution as follows. If using two dyes together, soak embryos in the combined dye solution for the longer of the two indicated durations.

- For LysoTracker Green (75 nM), soak embryos for $10 \mathrm{~min}$.

- For acridine orange $(1 \mu \mathrm{M})$, soak embryos for $5 \mathrm{~min}$.

- For MitoTracker Green (200 nM), soak embryos for 30 min. 
Downloaded from http://cshprotocols.cshlp.org/ on April 26, 2023 - Published by

Fluorescent Cell Staining Methods for H. exemplaris Embryos

- For FM 4-64 (10 $\mu \mathrm{g} / \mathrm{mL})$, soak embryos for $30 \mathrm{~min}$.

See Troubleshooting.

8. Remove the embryos from the dye droplet with the aspirator pipette. Rinse the embryos in a separate plastic Petri dish with $2 \mathrm{~mL}$ of spring water for $10 \mathrm{~min}$. Keep away from light.

To rinse the dye from the embryos, swirl the Petri dish and use the aspirator pipette to move embryos through the dish.

Proceed to Step 15.

9. After completion of the experiment, discard the used glass pipette, as dye accumulates inside and can contaminate other cultures and experiments.

Mark Nuclei with PI by Electroporation

Pl can be used in this electroporation protocol to mark nuclei.

10. Prepare PI staining solution $(10 \mu \mathrm{M})$. Pipette $15 \mu \mathrm{L}$ into a $0.4-\mathrm{cm}$ electroporation cuvette.

The liquid must be touching both metal sides of the cuvette.

11. Using an aspirator pipette, transfer 5-15 isolated embryos to the cuvette.

Transfer as little water as possible with the aspirator pipette to minimize dilution. 12-15 $\mu \mathrm{L}$ is sufficient for staining 5-15 embryos at a time.

12. Electroporate the cuvette at $0.5 \mathrm{kV}, 200 \Omega$, and $25 \mu \mathrm{FD}$ with three pulses, spaced apart by $30 \mathrm{sec}$. In our hands, these were the optimal settings to minimize embryo death and to maximize dye introduction. We have also had success introducing PI using both higher voltage with lower resistance and lower voltage with higher resistance; these conditions may be considered when trying to introduce other molecules into embryos.

13. Remove the embryos from the cuvette with the aspirator pipette. Rinse the embryos in a separate plastic Petri dish with $2 \mathrm{~mL}$ of spring water for $10 \mathrm{~min}$. Keep away from light.

To rinse the dye from the embryos, swirl the Petri dish and use the aspirator pipette to move embryos through the dish.

It is not unusual to lose some embryos at this stage. To recover more of the embryos, pipette additional spring water into the small amount of liquid left in the cuvette, pipette gently to mix, and transfer the liquid into the Petri dish.

Proceed to Step 15.

14. After completion of the experiment, discard the used glass pipette, as dye accumulates inside and can contaminate other cultures and experiments.

Mount Embryos on Slides

We use microscopic glass beads as precisely sized spacers to support the coverslip near the height of the embryos, and we seal the coverslip with Valap, which is gas-permeable, to prevent evaporation. We keep Valap warmed at $68^{\circ} \mathrm{C}$ in a glass test tube containing a paintbrush at all times.

15. Place a small drop of spring water (about $10 \mu \mathrm{L})$ on the center of a glass slide.

16. Using an aspirator pipette, transfer one or more embryos (no more than four is recommended) to the center of the droplet.

Placing too many embryos near each other can make imaging of each embryo difficult, as dyes in some embryos may photobleach before they are imaged.

17. Dip a clean micropipette tip into a vial of glass beads, and tap the vial to remove the excess beads from the tip. Tap the coated pipette tip on the inside edges of the water droplet to transfer the beads.

18. Carefully place a coverslip on top of the droplet. If the space under the coverslip is not entirely filled with water, pipette more spring water onto the edge of the coverslip and allow the water to spread. 
K.M. McGreevy et al.

19. Seal the coverslip edges with Valap by painting each side with a single stroke of the paintbrush, adding extra Valap to fill holes if necessary.

Holes can be seen under a stereomicroscope.

20. Locate the embryo(s) under a stereomicroscope and circle them with a marker on the slide (not on the coverslip, as objective oil may dissolve the ink).

Perform Imaging

H. exemplaris was chosen as a model system in part because animals and embryos are optically clear at all stages, facilitating the visualization of events in living material (Gabriel et al. 2007). It is useful to familiarize oneself with $\mathrm{H}$. exemplaris embryo autofluorescence at different stages. Most embryos have low-level autofluorescence, but gut granules fluoresce brightly. The eggshell has low-level autofluorescence. We use confocal microscopy or spinning disk confocal microscopy to visualize fluorescence. We check multiple channels to ensure that the patterns we see are not appearing in channels we do not expect based on the dyes we're using.

21. Place the slide on a microscope under DIC optics. Use a 10× objective to find and center the embryos. Use a $60 \times$ oil objective for imaging.

See Troubleshooting.

22. Change the dichroic mirror and filters to match the appropriate wavelength for each dye. Start with low laser power and increase as needed for imaging.

See Troubleshooting.

23. Film at room temperature.

See Troubleshooting.

Problem (Step 7): The staining solution dries up during staining.

Solution: Keep the Petri dish used for soaking in a humidity chamber and/or cover the Petri dish. If a 96-well plate is used and liquid is evaporating from the wells, add water to the adjacent wells to increase the local humidity.

Problem (Step 21): Embryos are not found under the confocal microscope.

Solution: This can happen if the embryos end up under the Valap or if the field of view on the microscope is too small to easily find the embryos. Return the objective to $10 \times$ to find the embryos and center them before returning to the $60 \times$ lens.

Problem (Steps 21-23): Embryos move as the objective lens is lowered and during filming.

Solution: This happens if the preparation is too thick, if the slide slowly dries out during filming, or if the embryos are mounted too close to the edge of the coverslip. Avoid a thick preparation by using slightly smaller glass beads and/or placing less water on the slide. Avoid drying by checking that the Valap seal is complete. If the embryos are mounted too close to the edge of the coverslip, then while imaging the objective lens may come into contact with the Valap lining the edges, causing an uneven downward force on the coverslip. Avoid this by placing the embryos in the center of the coverslip area, and take care to minimize the thickness of Valap added.

Problem (Step 22): Organelles are not fluorescing.

Solution: This can happen if the staining solution is old or overly dilute. Avoid using old solution by checking the solution date; many dyes are stable for only $\sim 6$ mo in stock solution. Avoid freezethaw cycles of your solution to prevent degradation. Try increasing the concentration of solution 


\title{
DISCUSSION
}

used or using fresh solution. Also, we have sometimes had more success using newly laid embryos (near the 1- to 4-cell stage) than with using older embryos, suggesting that under some conditions eggshells may become impermeable over time. Therefore, try using early-stage embryos if later stage embryos are not staining. For propidium iodine staining, we often find that nuclei become more well marked several hours to a day after staining.

Problem (Step 22): Organelles are fluorescing in more channels than expected.

Solution: The aspirator pipette tip can retain enough dye to stain other embryos that it contacts. We change the pipette tip between experiments to avoid contamination.

\begin{abstract}
Certain other live-cell dyes and fluorescent materials have given only limited success to date: SiR actin and SiR tubulin (Cytoskeleton Inc), and Sytox Green (Molecular Probes). In our experience, neither $\mathrm{SiR}$ actin or SiR tubulin labeled the expected structures in embryos when introduced via electroporation, soaking, or microinjection into adults. Sytox Green (a membrane-impermeant nucleic acid dye) localized as expected in embryos after microinjection of gravid mothers, but we do not typically use Sytox Green because electroporation with PI is easier than injection and results in a brighter signal.

Given the relative ease of each protocol that we tested, in general we recommend the following progression of protocols when attempting to introduce other dyes: soaking early embryos, electroporation, and microinjection of gravid animals. Microinjection of adults also works for RNAi even when eggs are not directly injected (Tenlen et al. 2013), suggesting that eggs can take up nucleic acids introduced elsewhere in the mother's body.
\end{abstract}

\section{RECIPES}

Acridine Orange Staining Solution (1 $\mu \mathrm{M})$

1. Prepare a stock solution of $1 \mathrm{~mm}$ acridine orange (Invitrogen A1301) in moleculargrade $\mathrm{H}_{2} \mathrm{O}$. Cover with aluminum foil to minimize light exposure. Store at room temperature.

2. Immediately before use, dilute the stock solution with spring water to prepare a staining solution of $1 \mu \mathrm{m}$ acridine orange.

\section{FM 4-64 Staining Solution $(10 \mu \mathrm{g} / \mathrm{mL})$}

1. Dissolve one vial $(100 \mu \mathrm{g})$ of FM 4-64 (Invitrogen T13320) in $100 \mu \mathrm{L}$ of spring water to prepare a stock solution of $1000 \mu \mathrm{g} / \mathrm{mL}$. Cover with aluminum foil to minimize light exposure. Store at or below $-20^{\circ} \mathrm{C}$.

2. Immediately before use, dilute the stock solution with spring water to prepare a staining solution of $10 \mu \mathrm{g} / \mathrm{mL}$ FM 4-64.

\section{LysoTracker Green DND-26 Staining Solution (75 nм)}

1. Allow a vial of $1 \mathrm{~mm}$ LysoTracker Green DND-26 solution (50 $\mu \mathrm{L}$ in DMSO) (Invitrogen L7526) to warm to room temperature. Briefly centrifuge to deposit the solution at the bottom of the vial before opening.

2. From the $1 \mathrm{~mm}$ solution, prepare a $500 \mathrm{~nm}$ stock solution using molecular-grade $\mathrm{H}_{2} \mathrm{O}$. Cover in aluminum foil to minimize light exposure. Store at or below $-20^{\circ} \mathrm{C}$.

3. Immediately before use, prepare a staining solution of roughly 75 пм Lysotracker Green by combining $2 \mu \mathrm{L}$ of the $500 \mathrm{~nm}$ stock solution with $10 \mu \mathrm{L}$ of spring water. 
K.M. McGreevy et al.

\section{MitoTracker Green FM Staining Solution (200 пм)}

1. Dissolve one vial $(50 \mu \mathrm{g})$ of MitoTracker Green FM (Invitrogen M7514) in DMSO to prepare a stock solution of $1 \mathrm{~mm}$. Cover with aluminum foil to minimize light exposure. Store at or below $-20^{\circ} \mathrm{C}$.

2. Immediately before use, dilute the stock solution with spring water to prepare a staining solution of $200 \mathrm{~nm}$ MitoTracker Green FM.

Propidium lodide (PI) Staining Solution $(10 \mu \mathrm{M})$

1. Prepare a $1 \mathrm{~mm}$ stock solution of propidium iodide (PI) (Invitrogen P3566; $1.0 \mathrm{mg}$ / $\mathrm{mL}$ ) in $\mathrm{H}_{2} \mathrm{O}$. Cover in aluminum foil to minimize light exposure. Store at $2^{\circ} \mathrm{C}-8^{\circ} \mathrm{C}$.

2. Immediately before use, dilute the stock solution with spring water to prepare a staining solution of $10 \mu \mathrm{M}$ PI.

Valap Sealant

Add equal weight of petroleum jelly (e.g., Vaseline), lanolin, and paraffin together in a 1-L beaker. (The preparation of a few hundred milliliters of this mixture should be a lifetime supply.) Heat the mixture at a low setting on a hot plate. Stir occasionally until thoroughly blended. Take extreme care not to overheat this mixture. Warm it just enough to make the components liquefy. The final product should be golden yellow, the color of corn or canola oil. If it is dark brown, it was heated too much. In that case, start again. Aliquot the final mixture into several small screw-capped jars $(\sim 50-\mathrm{mL}$ capacity). Store at room temperature. Keep a small amount in a $25-\mathrm{mL}$ beaker, and warm at a low setting on a hot plate before use.

\section{ACKNOWLEDGMENTS}

Support for the laboratory's work with live imaging of tardigrade embryos has been provided by National Science Foundation (NSF) grants IOS 0235658, IOS 1257320, and IOS 1557432.

Author contributions: K.M.M. and B.G. designed the experiments. K.M.M. developed the protocols for marking lysosomes, mitochondria, and nuclei by soaking or electroporation. K.L.H., S.K., and M.E.T. established that the soaking protocol also works for marking membranes. K.M.M. wrote the manuscript with input from the other authors.

\section{REFERENCES}

Gabriel WN, McNuff R, Patel SK, Gregory TR, Jeck WR, Jones CD, Goldstein B. 2007. The tardigrade Hypsibius dujardini, a new model for studying the evolution of development. Dev Biol 312: 545-559.

Lin Y, Lin J, Tsai T, Chen H, Chou K, Yang S, Tang Y, Hwang T. 2017. Acridine orange exhibits photodamage in human bladder cancer cells under blue light exposure. Sci Rep 7: 14103.
McNuff R. 2018. Laboratory culture of Hypsibius exemplaris. Cold Spring Harb Protoc doi:10.1101/pdb.prot102319.

Tenlen JR, McCaskill S, Goldstein B. 2013. RNA interference can be used to disrupt gene function in tardigrades. Dev Genes Evol 223: 171-181. 


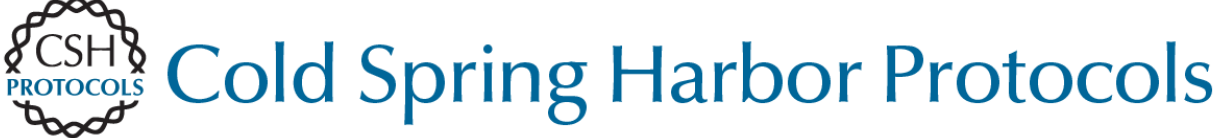

\section{Fluorescent Cell Staining Methods for Living Hypsibius exemplaris Embryos}

Kristen M. McGreevy, Kira L. Heikes, Shiri Kult, Marla E. Tharp and Bob Goldstein

Cold Spring Harb Protoc; doi: 10.1101/pdb.prot106021

\begin{tabular}{|c|c|}
\hline $\begin{array}{r}\text { Email Alerting } \\
\text { Service }\end{array}$ & Receive free email alerts when new articles cite this article - click here. \\
\hline $\begin{array}{l}\text { Subject } \\
\text { Categories }\end{array}$ & $\begin{array}{l}\text { Browse articles on similar topics from Cold Spring Harbor Protocols. } \\
\text { Confocal Microscopy ( } 114 \text { articles) } \\
\text { Developmental Biology (728 articles) } \\
\text { Emerging Model Organisms (321 articles) } \\
\text { Fluorescence (517 articles) } \\
\text { Fluorescence, general ( } 341 \text { articles) } \\
\text { Imaging Development ( } 255 \text { articles) } \\
\text { In Vivo Imaging (334 articles) } \\
\text { In Vivo Imaging, general (168 articles) } \\
\text { Labeling for Imaging (339 articles) }\end{array}$ \\
\hline
\end{tabular}

